\title{
A layered cooling process in whole quenching process for lightweight front axle beam
}

\author{
Kai Gao, Xunpeng Qin a , Zhou Wang, Shengxiao Zhu, Zhenyu Yuan, and Zhenhua Zhu \\ Hubei Key Laboratory of Advanced Technology for Automotive Components, Wuhan University of \\ Technology, Wuhan, Hubei 430070, China
}

\begin{abstract}
In order to improve the uniformity of martensite distribution and obtain consistent mechanical performance during the quenching process of front axle beam, a layered cooling process was presented in this paper. The fluid-solid coupled temperature field were investigated by finite element modeling and the prediction of micro-structure and hardness distributions were also carried out. The experimental result verifies the reliability of this model.
\end{abstract}

\section{Introduction}

Front axle is one of the most important parts involving safety in truck, and it is also the most complicated and heavy forging parts, which is used to carry the weight of the front part of the vehicle [1, 2]. During the using process, front axle beam is requested a proper strength and impact toughness because of the alternate-complicated stress on front axle beam, which also indicates a high technology requirement in thermal treatment. In order to realize the light-weight of front axle beam [3, 4], the center zone has several holes. Figure 1 shows the photograph of the front axle beam. It can be seen that the front axle thickness decreases from center to both ends. The thermal treatment process of front axle beam consist of quenching [5, 6] and tempering [7, 8]. Quenching process is to obtain the martensite structure and tempering process is to obtain the tempered sorbite structure. In the traditional quenching process, the front axle beam was heated to $840^{\circ} \mathrm{C}$, and then put into the quenching medium polyalkylene glycol (PAG) and cooled to room temperature. Due to the relatively great thickness in both ends of front axle beam, the percentage of martensite in core is lower than the center after quenching process. As a result, the strength does not reach the technology requirement after thermal treatment process. In order to improve the uniformity of martensite distribution and mechanical properties, a layered cooling process was carried out during the quenching process, which indicates different cooling process are adopted in different position of front axle beam. Modeling is a crucial elements in the design and optimization of thermal treatment systems [9]. However, modeling of layered cooling process is a challenging task since an appropriate model must deal with the following problems: multi-physics coupling, strong material non-linearity, complex boundary conditions and geometries. Among the above problems, multi-physics

\footnotetext{
${ }^{\text {a }}$ Corresponding author: qxp915@sina.com
}

This is an Open Access article distributed under the terms of the Creative Commons Attribution License 4.0, which permits unrestricted use, distribution, and reproduction in any medium, provided the original work is properly cited. 


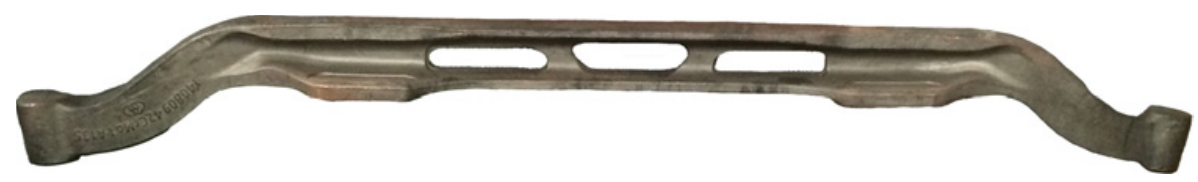

Figure 1. Photograph of the front axle beam.

(a)

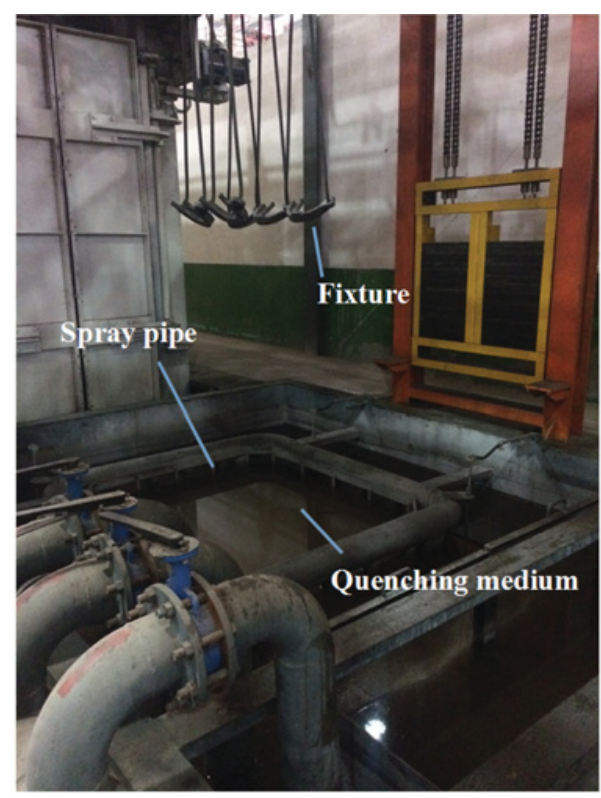

(b)

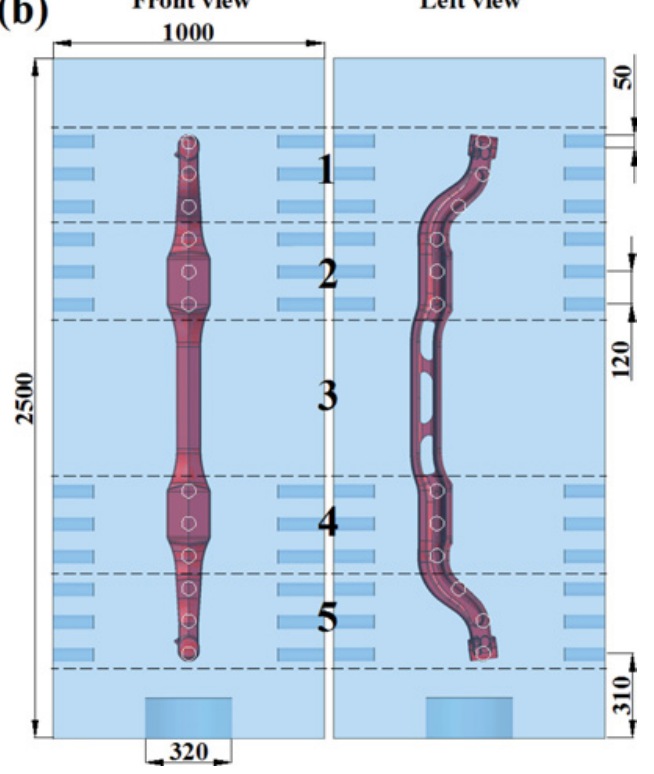

Figure 2. Schematic diagram of layered cooling process. (a) Photograph of equipment, (b) Physical model.

coupling including fluid-solid, temperature and phase transformation makes the modeling process more difficult.

The present work is focused on improving the consistent mechanical performance, especially the micro-structure and hardness uniformity of a truck front axle beam. A 3D model coupled fluid-solid, temperature and phase transformation fields was built by a finite element software. The temperature evolution and prediction of micro-hardness and micro-structure distributions were carried out.

\section{Technical principle of the process}

During the quenching process, how to guarantee the micro-structure and hardness uniformity of front axle beam with complex shape and large size is one of the difficult problems. In order to optimize this process, the layered cooling process was carried out. Figure 2 shows the schematic diagram of layered cooling process. It can be obviously seen that the front axle beam are divided to five parts for cooling. As front axle beam is fixed by fixture and put into the quenching medium completely, the cooling medium will be spraying on it at the same time. Spray pipe are installed in the quenching layer of parts 1, 2, 4 and 5 and each layer is installed twelve spray pipe from four direction in total (three in each direction), the relative position with front axle beam is shown in Fig. 2(b). In addition, the spraying velocity of cooling medium in layers 1 and 5 is larger than that in layers 2 and 4 according to the thickness of front axle beam. The bottom surface of quenching flume is outlet. 
Table 1. Parameters in the layer cooling process.

\begin{tabular}{|c|c|}
\hline Item & Parameter \\
\hline Workpiece material & AISI 4140 steel \\
\hline Total cooling time (s) & 100 \\
\hline Quenching medium & $5 \%$ PAG \\
\hline Spraying velocity (layers 1 and 5) (m/s) & 5 \\
\hline Spraying velocity (layers 2 and 4) (m/s) & 3 \\
\hline
\end{tabular}

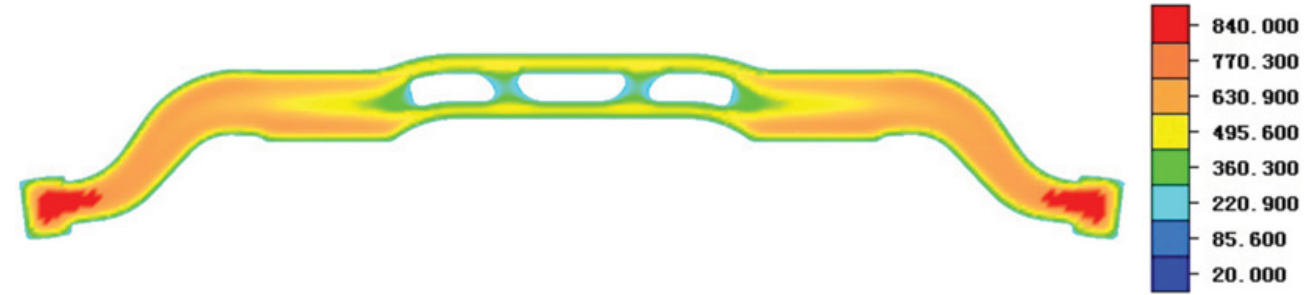

Figure 3. Temperature cloud map of front axle beam center cross-section under traditional process at $20 \mathrm{~s}$.

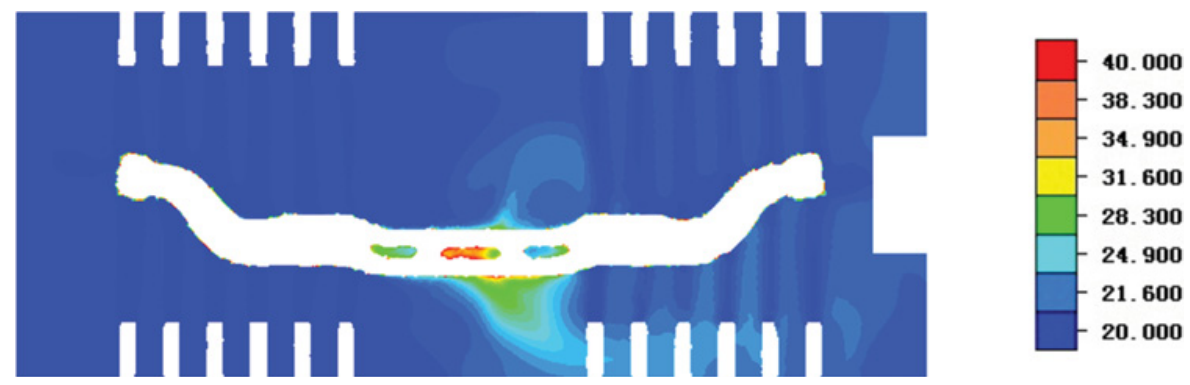

Figure 4. Temperature cloud map of quenching flume under layered process at $20 \mathrm{~s}$.

According to the technology requirement of thermal treatment process, the tensile strength is constrained between 880 and $1030 \mathrm{MPa}$ after tempering process. The basic information of simulation is shown in Table 1 according to the parameters of thermal treatment equipment in the quenching process.

\section{Results and discussion}

\subsection{Temperature field results}

In order to optimize the quenching process of truck front axle beam, the traditional cooling process was simulated by FE modeling and the total time is $100 \mathrm{~s}$. Figure 3 shows temperature cloud map of front axle beam center cross-section under traditional process at $20 \mathrm{~s}$. It can be obviously seen that the temperature around the holes and surface is lower that other parts of front axle beam, which indicates the cooling rate is relatively large in these parts. Due to the different thickness of front axle beam, the core of front axle beam each end keeps relatively high temperature and decreases gradually to the center. Therefore, the traditional cooling process leads to a large non-uniform cooling rate distribution on the whole front axle beam and has a negative effect on the following tempering process.

Figure 4 shows the temperature cloud map of quenching flume under layered process at $20 \mathrm{~s}$. It can be seen that a relatively large temperature gradient of cooling medium appears in the center of quenching flume. This is because no spraying pipe are set in this zone, which leads to a relatively low flow velocity 
(a)

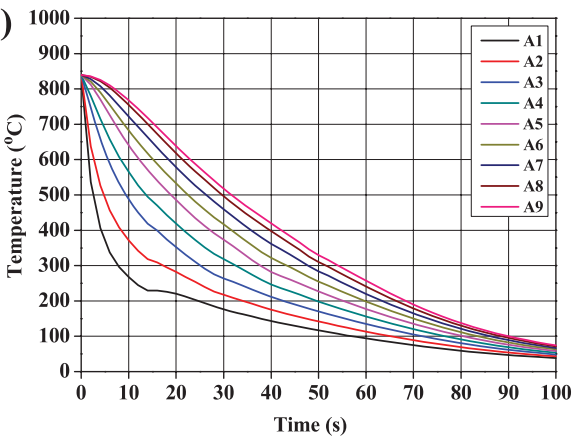

(c)

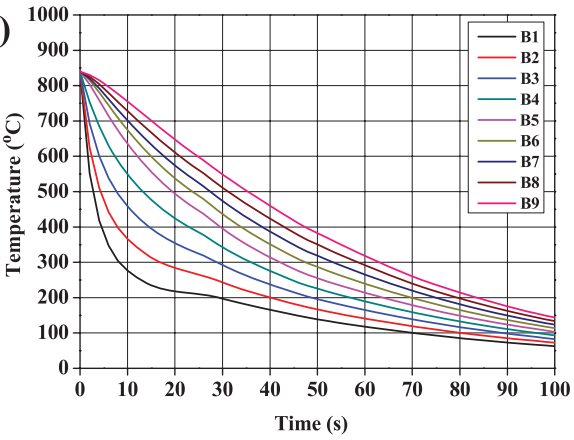

(b)

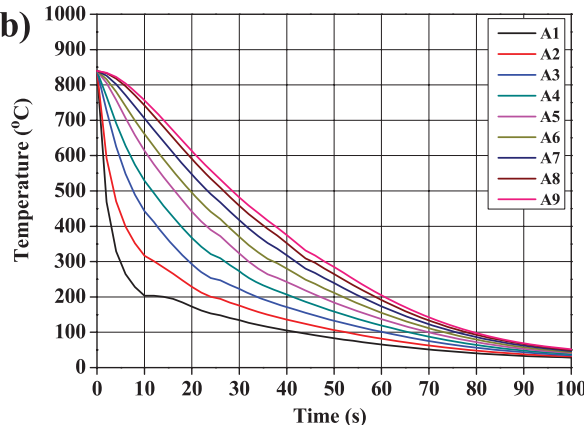

(d)

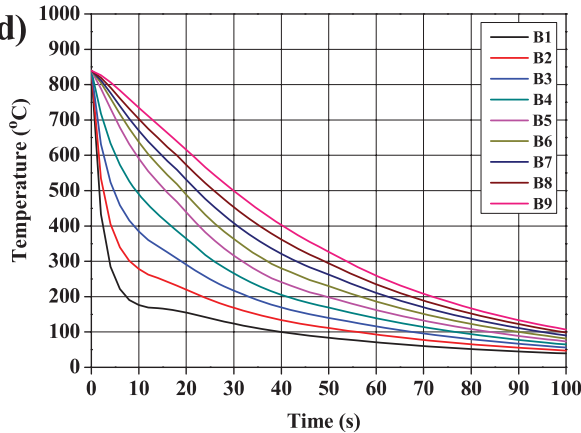

(e)

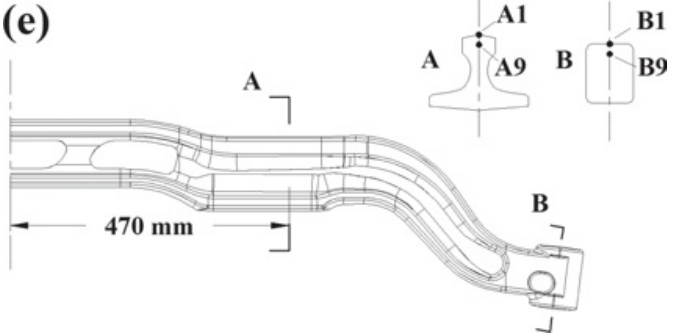

Figure 5. Time evolution of the temperature at the selected points on the cross-section center line. (a) Traditional process of section A, (b) Layered process of section B, (c) Traditional process of section A, (d) Layered process of section B, (e) Relative position of section A and B.

of cooling medium in center zone. The highest temperature is below $40^{\circ} \mathrm{C}$, it indicates that the cooling medium in the quenching flume has a large flow velocity as a whole.

In this simulation, the cross-section $\mathrm{A}$ and $\mathrm{B}$ were selected to compare the difference between the traditional and layered cooling process, which is shown in Fig. 5(e). Figure 5(a) and (c) show time evolution of the temperature at the selected points on the cross-section A and B center line under the traditional cooling process while Fig. 5(b) and (d) show that under the layered cooling. Points A1 and B1 is on the surface, and then points A2 to A9 (points B2 to B9) are selected in the depth direction by number order. The least distance between each point is $2 \mathrm{~mm}$. It can be seen in Fig. 5 that cooling rate of each points becomes lower with time and also lower with the increment of the distance from surface. Compared with Fig. 5(a) and (c), as part of section A is covered by spray cooling medium, the cooling rate of points A1 to A9 all become larger and the increment of cooling rate decreases with increment of 


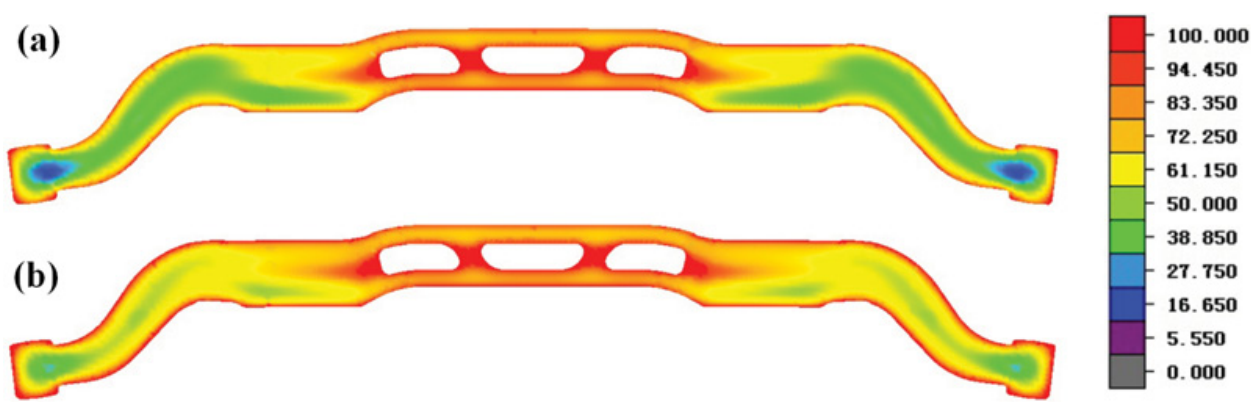

Figure 6. Martensite distribution of center cross-section front axle beam at 100s. (a) Traditional cooling process, (b) Layered cooling process.

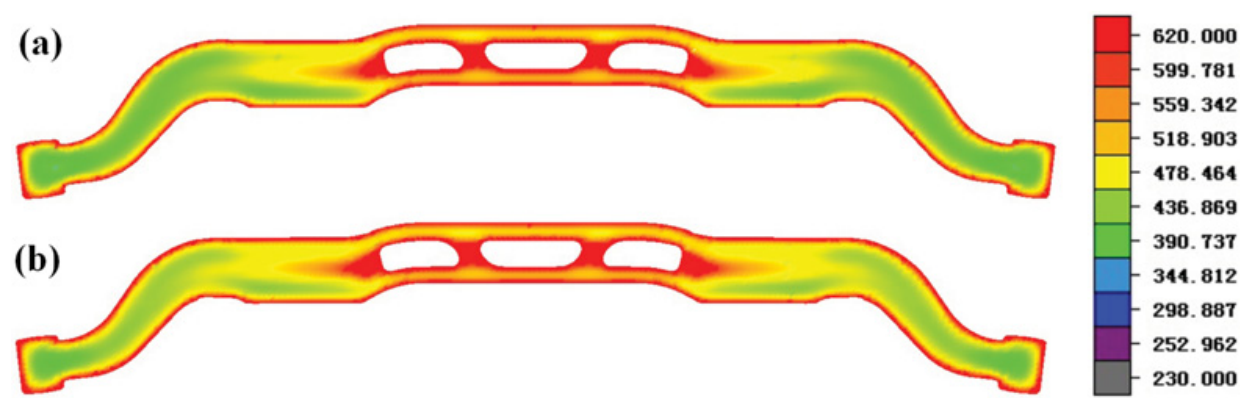

Figure 7. Hardness distribution of front axle beam center cross-section at 100s. (a) Traditional cooling process, (b) Layered cooling process.

the distance from surface. Compared with Fig. 5(b) and (d), the similar variation also appears on section $\mathrm{B}$ and the cooling rate increment of points B1 to B9 is larger that that of points A1 to A9 at the same distance from surface.

\subsection{Prediction of phase transformation}

The simulation of phase transformation is based on the continual cooling transformation (CCT) curves of AISI 4140 steel, which is used in track front axle beam. Martensite distribution of front axle beam center cross-section at 100s under traditional and layered cooling process are shown in Fig. 6(a) and (b), respectively. It can be obviously seen that the percentage of martensite is $100 \%$ around the holes and on the surface, the core of front axle beam each end shows the lowest percentage of martensite. Compared with Fig. 6(a) and (b), the martensite percentage of the whole front axle beam increases after the layered cooling process, which indicates the uniformity of martensite distribution is improved.

Figure 7(a) and (b) shows hardness distribution of front axle beam center cross-section at 100s under traditional and layered cooling process, respectively. It can be obviously seen that the hardness value of whole front axle beam increases after the layered cooling process and the uniformity of hardness distribution is also improved.

As mentioned above in Sect. 2, the thermal treatment equipment is shown in Fig. 2(a) and the basic information is shown in Table 1. During the experiment process, sections A and B were chosen to measure the mechanical performance, especially the tensile strength. The experimental result shows that the tensile strength in those two parts reach the technology requirement, which also indicates that 


\section{MATEC Web of Conferences}

the layered cooling process can improve the mechanical performance of front axle beam during the quenching process. Therefore, the reliability of this model was verified by the experimental results.

\section{Conclusion}

This paper proposes a finite element model of layered cooling process on truck front axle beam of AISI 4140 steel. The following conclusion have been reached:

(1) The FE model coupled fluid-solid, temperature and phase transformation fields was built. Temperature field of front axle beam and quenching flume were presented and the prediction of micro-structure and hardness distributions were carried out.

(2) The truck front axle beam quenching process is optimized and the uniformity of martensite distribution is improved. The experiment result verifies the reliability of this model.

The financial support of the National Natural Science Foundation of China (NSFC) (No. 51175392) and the Key Projects of Hubei Province Science \& Technology Pillar Program (No. 2014BAA012) are gratefully acknowledged. The authors are grateful to all the staff of Hubei Key Laboratory of Advanced Technology for Automotive Components for supporting this work.

\section{References}

[1] R. Li, S. Jiao, J. Wang, IERI Procedia, 1 (2012)

[2] G.D. Satish, N.K. Singh, R.K. Ohdar, J. Mater. Process. Technol., 203 (2008)

[3] M. Duraiselvam, A. Valarmathi, S.M. Shariff, G. Padmanabham, Wear, 309 (2014)

[4] E. Schubert, M. Klassen, I. Zerner, C. Walz, G. Sepold, J. Mater. Process. Technol., 115 (2001)

[5] C. Şimşir, C. Hakan Gür, J. Mater. Process. Technol., 207 (2008)

[6] S.K. Dhua, S.K. Sen, Mat. Sci. Eng. A-Struct., 528 (2011)

[7] S. Kahrobaee, M. Kashefi, J. Magn. Magn. Mater., 382 (2015)

[8] G. Chakraborty, C.R. Das, S.K. Albert, A.K. Bhaduri, V. Thomas Paul, G. Panneerselvam, A. Dasgupt, Mater. Charact., 100 (2015)

[9] C. Şimşir, C. Hakan Gür, Comp. Mater. Sci., 44 (2008) 